\title{
Procesos de Visualización en una Tarea de Generación y Representación de Cuerpos de Revolución
}

\author{
Visualization Processes in a Task that Requires Generating and \\ Representing Solids of Revolution
}

\author{
Teresa F. Blanco* \\ ORCID iD 0000-0003-4215-8677 \\ Jose Manuel Diego-Mantecón ${ }^{* *}$ \\ ORCID iD 0000-0002-4427-2724 \\ Pablo González Sequeiros ${ }^{* * *}$ \\ ORCID iD 0000-0001-9486-814X
}

\begin{abstract}
Resumen
En este trabajo se analizaron los procesos de visualización implicados en una tarea que requiere generar y representar cuerpos de revolución. La tarea se administró a cuatrocientos estudiantes del grado de maestro de Educación Primaria. Se adoptó como marco teórico el enfoque Ontosemiótico de la Instrucción y el Conocimiento Matemáticos, utilizándose como herramienta analítica la noción de configuración en su vertiente epistémica y cognitiva. Para el análisis de datos se empleó una metodología de investigación mixta: con una componente cualitativa que evaluó las categorías de objetos primarios, y otra cuantitativa que categorizó el grado de corrección de las respuestas. El análisis de los procesos de visualización se realizó por medio de las nueve configuraciones cognitivas identificadas en las soluciones de los estudiantes. Los errores de resolución se concentraron más en el procesamiento visual de la información que en el proceso de interpretación de la información figurativa. La mayoría de ellos se debieron a dificultades para movilizar las habilidades de reconocimiento de relaciones y posiciones espaciales, observándose una correspondencia entre la efectividad de las configuraciones cognitivas y la representación externa de los cuerpos generados.
\end{abstract}

Palabras clave: Visualización. Cuerpos de revolución. Representación plana. Enfoque Ontosemiótico. Formación de maestros.

\begin{abstract}
In this work we analyzed the visualization processes involved in a mathematical task that requires generating and representing solids of revolution. The task was administered to four hundred university students from the

\footnotetext{
* Doctora en Matemáticas por la Universidade de Santiago de Compostela (USC). Profesora Titular en la Universidade de Santiago de Compostela (USC), Santiago de Compostela, España. Dirección Postal: Facultade de Ciencias da Educación // Av. Xoán XXIII, s/n, CP: 15782, Santiago de Compostela, A Coruña, España. E-mail: teref.blanco@usc.es.

${ }^{* *}$ Doctor en Educación Matemática por la University of Cambridge. Profesor Ayudante Doctor en la Universidad de Cantabria (UC), Santander, España. Dirección Postal: Facultad de Ciencias // Av. De los Castros, 48, CP: 39005, Santander, España. E-mail: diegojm@unican.es.

**** Doctor en Matemáticas por la Universidade de Santiago de Compostela (USC). Profesor Contratado Doctor en la Universidade de Santiago de Compostela (USC), Lugo, España. Dirección Postal: Facultade de Formación do Profesorado // Av. Ramón Ferreiro, s/n, CP: 27002, Lugo, España. E-mail: pablo.gonzalez.sequeiros@ usc.es.
} 
Bachelor's Degree in Primary Education. We adopted the Ontosemiotic Approach to Mathematical Instruction and Knowledge as a theoretical framework, using the configuration notion as an analytical tool in both its epistemic and cognitive aspects. For the data analysis, we used a mixed method approach: a qualitative dimension evaluating the primary objects categories, and a quantitative dimension categorizing the answers' success degree. The visualization processes analysis was done via the nine cognitive configurations identified from the students' solutions. The resolution errors focused more on the visual processing than on the interpreting figurative information processing. The majority of these errors were due to difficulties in mobilizing the recognition's abilities of spatial relations and positions, observing a correspondence between the effectiveness of cognitive configurations and the external representation of the generated solids.

Keywords: Visualization. Solids of revolution. Planar representation. Ontosemiotic Approach. Teacher training.

\section{Introducción}

La visualización es una componente clave del razonamiento matemático, fundamental en procesos de resolución de problemas, argumentación y prueba de diversas áreas de las matemáticas (CLEMENTS, 2014; LUBINSKI, 2010). En el caso particular de la geometría, adquiere un papel fundamental al tener esta un soporte muy fuerte en elementos visuales.

Según Bishop (1989) hay dos procesos implicados en la visualización que se encargan de regular y gestionar la conexión entre la información externa que percibe el estudiante y la información interna que genera como consecuencia de su actividad mental. El primero es el procesamiento visual de la información (VPI) que convierte la información externa, que puede ser gráfica (esquemas, figuras, gráficos, objetos reales o virtuales) o verbal (texto escrito o información oral), en imágenes mentales y, además, transforma unas imágenes ya formadas en otras. El segundo proceso, inverso al anterior, es el que este autor denomina interpretación de la información figurativa (IFI), que permite analizar una imagen mental y extraer información de ella. Esta información puede expresarse externa o internamente, en particular a través de una nueva imagen.

Estos dos procesos se pueden desarrollar trabajando diferentes representaciones planas de objetos tridimensionales y las relaciones entre estas (GAULIN, 1985; GUTIÉRREZ, 1998b). En las representaciones planas de los cuerpos tridimensionales existe una pérdida de información que dificulta el análisis de las propiedades de estos objetos, así como su reconocimiento (PARZYSZ, 1988).

Para interpretar esas representaciones planas y poder reconstruir los cuerpos tridimensionales a partir de ellas, hace falta la aplicación de ciertos convencionalismos que son fundamentales para comunicar la información espacial (GUTIÉRREZ, 1996b). Como consecuencia de lo anterior, muchos estudios de visualización espacial se centran en la instrucción de modelos de representación - como por ejemplo los trabajos de Ben-Chaim, 
Lappan y Houang (1988); Guillén et al. (1992); Gutiérrez (1992); Kopelman y Vinner (1994) o Michelmore (1980b, 1983).

Además, es necesario identificar etapas de evolución en la habilidad para dibujar formas tridimensionales, véanse por ejemplo Hazama y Akai (1993), Hershkowitz, Parzysz y Dormolen (1996), Michelmore (1980a), Pittalis, Mousoulides y Christou (2009).

En el caso concreto de los cuerpos de revolución no se encuentran investigaciones específicas encaminadas a la instrucción de modelos de representación, ni tampoco se han identificado, de manera explícita, niveles de habilidad en el dibujo de estas figuras, salvo el de Cohen (2003) para sólidos tridimensionales en general.

Autores como Guillén, González y García (2009) han analizado libros de texto de Educación Primaria y Secundaria para concluir que la mayoría de las actividades que se proponen sobre sólidos de revolución se centran en la medición (cálculo de volúmenes y áreas) e identificación, trabajándose, rara vez, las representaciones planas, y la generación de estos sólidos a partir de curvas o figuras bidimensionales. Tampoco se hacen menciones a posibles convenios para su representación.

Así mismo, estudios recientes de evaluación del conocimiento del contenido con futuro profesorado de Educación Primaria - como los de Gonzato, Godino y Contreras (2011); Blanco (2011); Godino et al. (2016) - sugieren que estos estudiantes tienen conocimientos muy limitados sobre los sólidos de revolución, centrados en la identificación de los más habituales (cono, cilindro y esfera) y en el cálculo de áreas y volúmenes; y que los porcentajes más bajos los alcanzan en ítems relacionados con la generación de sólidos.

El objetivo de este trabajo es profundizar en el conocimiento de los futuros maestros, analizando los procesos de visualización que ponen en juego al resolver una tarea de generación y representación de sólidos de revolución. Este análisis permitirá conocer cómo generan dichos sólidos, las imágenes mentales que crean y el tipo de representación que utilizan para comunicar dichas imágenes. Así mismo, ayudará a delimitar las dificultades de estos estudiantes en relación con dichos procesos.

\section{Marco teórico}

El marco teórico seguido en este trabajo es el enfoque ontosemiótico del conocimiento y la instrucción matemáticos (EOS), que integra diferentes puntos de vista y nociones teóricas sobre el conocimiento matemático, su enseñanza y aprendizaje (GODINO; BATANERO; FONT, 2007), y centra el análisis de la actividad matemática, y de los procesos y componentes 
que intervienen en esta, en las prácticas que realizan las personas implicadas en dicha actividad (GODINO, 2002; GODINO; BATANERO; FONT, 2007). Entendemos que este marco teórico puede contribuir en el campo de la visualización aportando un punto de vista complementario (GODINO et al., 2012) para abordar cuestiones tales como:

- ¿Qué tipo de procesos y objetos se ponen en juego cuando se realizan tareas de visualización?

- ¿Por qué algunas tareas que requieren visualización implican una gran dificultad para algunos estudiantes?

En este trabajo se tomará como herramienta analítica del EOS la noción de configuración ontosemiótica, definida como la red de objetos y procesos que se ponen en juego en la realización de una determinada práctica matemática (FONT; GODINO; GALLARDO, 2013). Se consideran, aquí, dos niveles de objetos que emergen de esas prácticas matemáticas. El primer nivel lo componen los objetos que se observan en un texto matemático como son las tareas, lenguaje, conceptos, procedimientos, propiedades y argumentos.

Estos objetos se articulan de modo que las tareas motivan las definiciones, procedimientos y proposiciones, y todos ellos, a su vez, condicionan los argumentos y regulan el uso del lenguaje. El lenguaje, por su parte, expresa y soporta esas definiciones, procedimientos y proposiciones.

Por último, los argumentos, a través del lenguaje, justifican las definiciones, procedimientos y proposiciones utilizados para la resolución de la tarea. Los objetos del segundo nivel (facetas duales) surgen de las distintas maneras de ver, hablar, operar sobre los objetos del nivel anterior; nos referimos a objetos personales o institucionales, ostensivos o no ostensivos, unitarios o sistémicos, intensivos o extensivos, y a contenido o expresión. Cuando se analizan las respuestas de sujetos individuales, dichas configuraciones se consideran cognitivas, mientras que, si son las respuestas esperadas desde un punto de vista institucional, se las denomina epistémicas.

Una aproximación a la visualización desde este marco teórico lleva a considerar las prácticas como prácticas visuales si ponen en juego la visualización, siempre teniendo en cuenta que se producen constantes conversiones entre componentes visuales y analíticos en la resolución de estas. El componente visual puede jugar un papel clave en la comprensión de la naturaleza de la tarea y en el momento de hacer conjeturas, mientras que el componente analítico cobrará protagonismo en el momento de la generalización y la justificación de las soluciones a través de los argumentos (GODINO et al., 2013). En Godino et al. (2012) puede verse una delimitación de los objetos primarios para el caso concreto de la visualización. En el 
apartado 3.1 esto se ilustrará con la configuración epistémica de la tarea propuesta en este estudio.

\section{Metodología}

El análisis de los procesos de visualización que se va a presentar se ha realizado aplicando el primer nivel de objetos de la configuración ontosemiótica, tanto a la solución experta de referencia (configuración epistémica) como a las soluciones dadas por los estudiantes (configuraciones cognitivas). Los tipos de configuraciones cognitivas que se determinan emergen del análisis de las prácticas manifestadas por los sujetos que responden a una tarea, tratándose, por tanto, de un análisis cualitativo. Por otra parte, se evalúa el grado de corrección de las respuestas a dicha tarea que, como variable cuantitativa, se resume y analiza mediante técnicas estadísticas. Se ha adoptado por tanto un enfoque metodológico de tipo mixto (HART et al., 2009).

La tarea se seleccionó del instrumento de evaluación de visualización de Blanco (2011) y se aplicó a una muestra de cuatrocientos estudiantes del grado de maestro de Educación Primaria. Se trata de una tarea de generación de cuerpos de revolución, en la que se muestran dos figuras (un triángulo y una lúnula) y los ejes de rotación sobre los que deben rotar (Figura 1). Se presentó para ser respondida en papel y lápiz, y se complementó con entrevistas semiestructuradas y grabaciones en vídeo que permitieron registrar los gestos que ayudan a conformar las imágenes mentales que crean los estudiantes y que no se recogen de una forma clara en las representaciones planas o escritas que realizan.

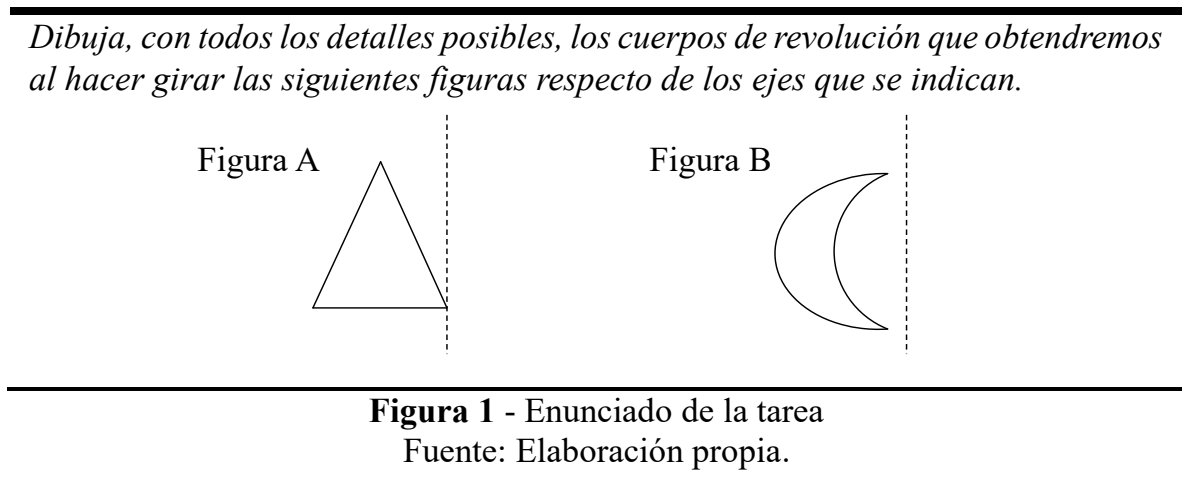

Estamos ante lo que Gonzato, Blanco y Godino (2011) categorizan como una tarea de interpretación de perspectivas de objetos tridimensionales, que contempla, entre otras, las dos acciones que se solicitan en la tarea: generar cuerpos de revolución y realizar una representación gráfica de dichos cuerpos. Como la respuesta es de dibujo, admite diferentes técnicas de representación (dibujo en perspectiva, vistas ortogonales etc.). Las figuras bidimensionales que 
se presentan no se pueden manipular físicamente, por lo que las acciones requeridas para resolver la tarea serán mentales. La tarea se sitúa por tanto como una práctica visual dentro del marco teórico adoptado (GODINO et al., 2012).

\subsection{Instrumento de análisis}

En el marco del EOS, el análisis de los procesos de visualización implicados en la resolución de la tarea requiere la elaboración de la configuración epistémica y las configuraciones cognitivas asociadas a dicha tarea.

\subsubsection{Configuración epistémica de la tarea}

La configuración epistémica nos permite explorar la tarea desde el punto de vista institucional, reconociendo las habilidades de visualización puestas en juego en la tarea y describiendo los objetos y procesos que se requieren para su resolución.

El primer objetivo que pretende la tarea es crear las imágenes mentales de los cuerpos de revolución generados al hacer girar las figuras A (triángulo) y B (lúnula) alrededor de los ejes dados; el segundo dibujar una representación plana de esos cuerpos. En su resolución se ponen en juego las tres habilidades de visualización siguientes (DEL GRANDE, 1987, 1990; GUTIÉRREZ, 1996a): reconocimiento de relaciones espaciales, rotación mental y reconocimiento de posiciones espaciales. Las dos primeras están implicadas sobre todo en el primer objetivo y la tercera en el segundo.

La habilidad de reconocimiento de relaciones espaciales establece la relación de cada una de las figuras con el eje de rotación, que determinará elementos internos de los cuerpos generados. También permite identificar correctamente las relaciones entre los diferentes elementos de cada una de las figuras. La habilidad de rotación mental es necesaria para producir las imágenes mentales del triángulo y la lúnula en movimiento.

La habilidad de reconocimiento de posiciones espaciales, a la hora de realizar la representación plana del cuerpo generado, tomando como punto de referencia una o varias de las posiciones del observador. Las habilidades de reconocimiento de relaciones y posiciones espaciales están estrechamente relacionadas en esta tarea, ya que deben ser integradas para hacer una representación plana que comunique la mayor información sobre los cuerpos generados.

El análisis del primer nivel de objetos de la tarea, descrito como un análisis semántico 
por Godino (2002), permitirá identificar el lenguaje, los conceptos, proposiciones, procedimientos y argumentos puestos en juego (Cuadro 1).

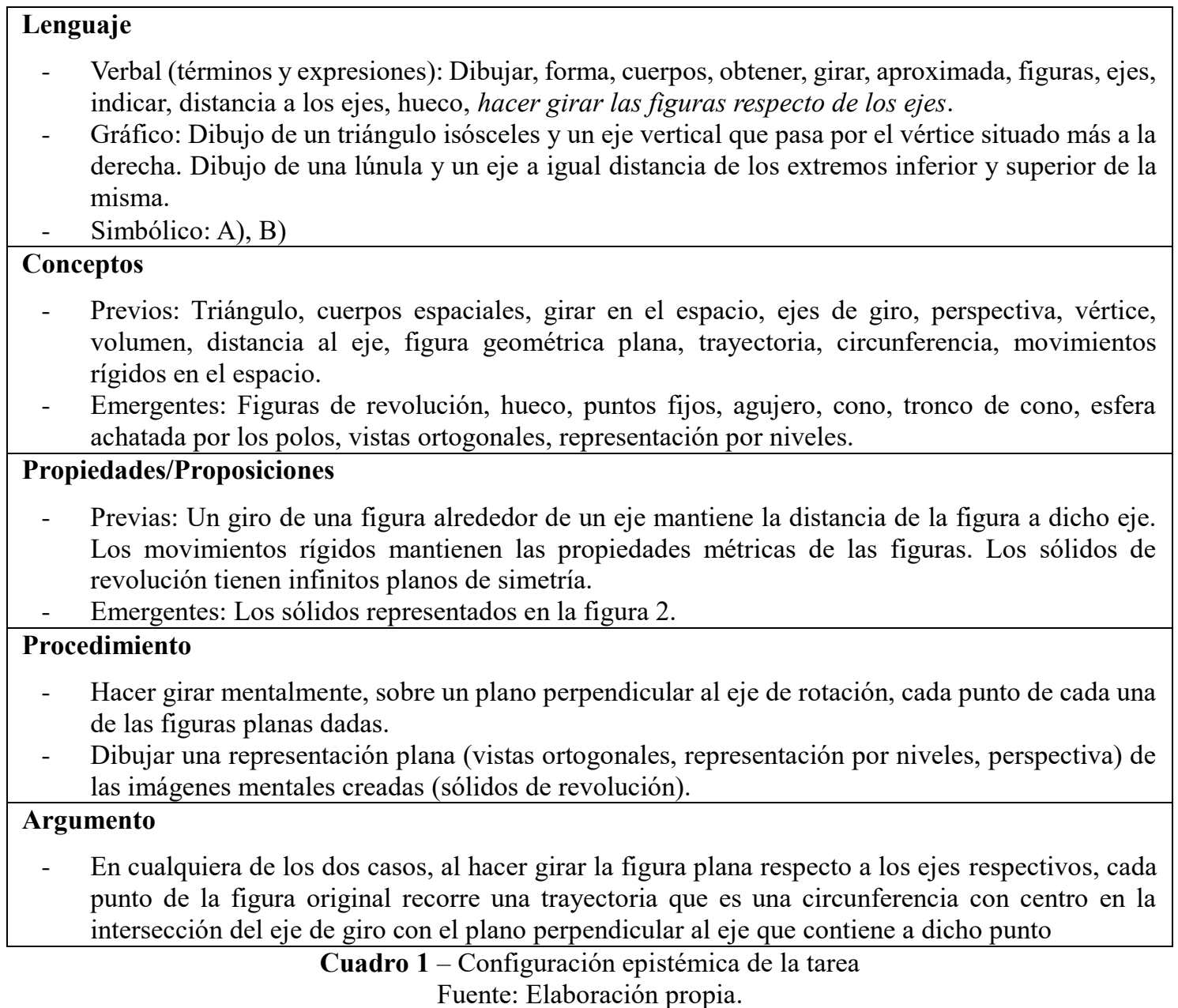

En el marco de EOS, las representaciones externas se consideran objetos ostensivos (cualquier objeto que es público y que, por tanto, se puede mostrar a otro) y las imágenes mentales objetos no ostensivos (no perceptibles por sí mismos). Estos objetos de segundo nivel dan lugar a los procesos de idealización y materialización (GODINO; FONT; WILHELMI, 2008) que se corresponden en el campo de la visualización con los procesos VPI e IFI, respectivamente.

Los objetivos pretendidos en la tarea ponen en juego esta faceta dual ostensivo/no ostensivo, de manera que el proceso VPI se requiere para interpretar la información externa que nos da la tarea (verbal en forma de texto escrito y gráfica en forma de una representación bidimensional), y el proceso IFI al solicitar como parte de la solución de la tarea una representación externa (gráfica en este caso) de la solución. En la Figura 2 se muestra, de forma esquemática, la resolución de la tarea desde los procesos implicados. 


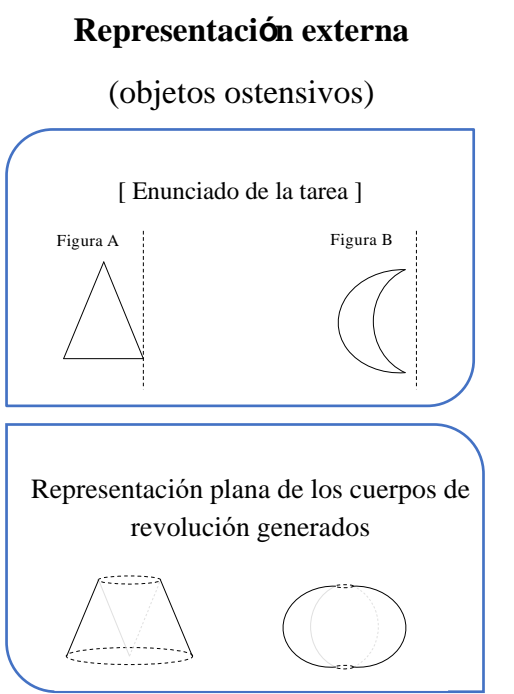

Imágenes mentales de la solución

(objetos no ostensivos)
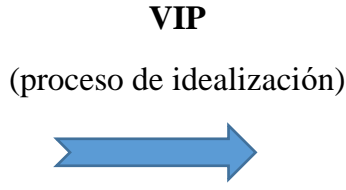

IFI

(proceso de materialización)

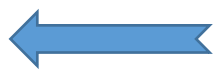

Figura A

Cono truncado con un hueco en forma de cono invertido
'Elipsoide achatado' con un agujero en forma de 'esfera achatada'

Figura 2 - Procesos de visualización en la solución epistémica de la tarea Fuente: Elaboración propia.

\subsubsection{Configuraciones cognitivas}

Para poder analizar los procesos de visualización que ponen en juego los estudiantes para maestro al resolver la tarea, se construyeron sus configuraciones cognitivas, identificando los objetos del primer nivel de forma similar al realizado para la configuración epistémica presentada en la sección anterior. El análisis permitió categorizar las configuraciones cognitivas (CC) de los cuatrocientos estudiantes, por medio de nueve configuraciones diferentes.

En la Tabla 1 se presentan estas configuraciones, que se nombran por el tipo de representación externa asociada a cada una de ellas y se enumeran en función de su porcentaje en las respuestas de los estudiantes. También se indica la dimensión de las imágenes generadas asociadas a cada una de las configuraciones. Por limitación de espacio, la descripción de cada una de las configuraciones se presentará en la siguiente sección, asociada al análisis del proceso IFI. Esta descripción solo incluirá, de forma resumida, los argumentos y el lenguaje gráfico aportado por los estudiantes para justificar la resolución de la tarea.

Tabla 1 - Configuraciones cognitivas ordenadas según el porcentaje de estudiantes que las manifiestan

\begin{tabular}{|c|c|c|c|c|c|}
\hline Configuración & $\begin{array}{l}\text { Dimensión de la } \\
\text { imagen generada }\end{array}$ & & Representación Externa & Frecuencia & $\%$ \\
\hline $\mathrm{CC} 1$ & $2 \mathrm{D}$ & \multirow{7}{*}{ Gráfica } & Simetría & 196 & 49,00 \\
\hline $\mathrm{CC} 2$ & $3 \mathrm{D}$ & & Perspectiva sin apoyo de los ejes & 66 & 16,50 \\
\hline $\mathrm{CC} 3$ & $2 \mathrm{D}$ & & Secuencia de giros en el plano & 23 & 5,75 \\
\hline $\mathrm{CC} 4$ & $3 \mathrm{D}$ & & Perspectiva con apoyo de los ejes & 21 & 5,25 \\
\hline $\mathrm{CC} 5$ & $3 \mathrm{D}$ & & Sección ortogonal-frontal & 21 & 5,25 \\
\hline $\mathrm{CC} 7$ & $3 \mathrm{D}$ & & Secuencia de giros en el espacio & 10 & 2,50 \\
\hline $\mathrm{CC} 8$ & $2 \mathrm{D}$ & & Composición giro-traslación & 9 & 2,25 \\
\hline
\end{tabular}




\begin{tabular}{|c|c|c|l|c|c|}
\hline CC9 & 3D & & Planta y alzado & 5 & 1,25 \\
\hline CC6 & 3D & Verbal & Escrita & 17 & 4,25 \\
\hline NC & \multicolumn{2}{|c|}{ No contesta } & 32 & 8 \\
\hline Total & \multicolumn{3}{|c|}{400} & 100 \\
\hline
\end{tabular}

Fuente: Elaboración propia.

\section{Resultados}

A continuación, se muestran los resultados del análisis de los dos procesos IFI y VPI que, combinados, darán cuenta de los cuerpos generados mentalmente por los estudiantes en la tarea propuesta. Se presentarán primero los relativos al proceso IFI, ya que es este el que aporta, a través de las representaciones externas, la principal evidencia que se tiene de cómo son las imágenes de los cuerpos generados por los estudiantes.

\subsection{Interpretación de la información figurativa}

En este estudio, la información viene expresada externamente de forma gráfica, a través de las representaciones planas de las imágenes de los cuerpos generados (al haberlo solicitado en la tarea), o de forma verbal, mediante la descripción escrita de dichas imágenes. El análisis de este proceso se presenta a través de la descripción de las nueve configuraciones cognitivas identificadas. En ellas se indica el tipo de imagen creada y la representación externa asociada.

CC1-Representación de una simetría. En esta configuración las imágenes mentales creadas son las figuras simétricas de las dadas. El procedimiento seguido consiste en tomar algunos puntos de las figuras y marcar sus simétricos respecto al eje dado. En el caso A los estudiantes tomaron los vértices del triángulo, en el B los vértices de la lúnula y algunos puntos de las curvas que la definen. En la Figura 3 se muestra como el estudiante 25 emplea este tipo de procedimiento para representar su solución. 

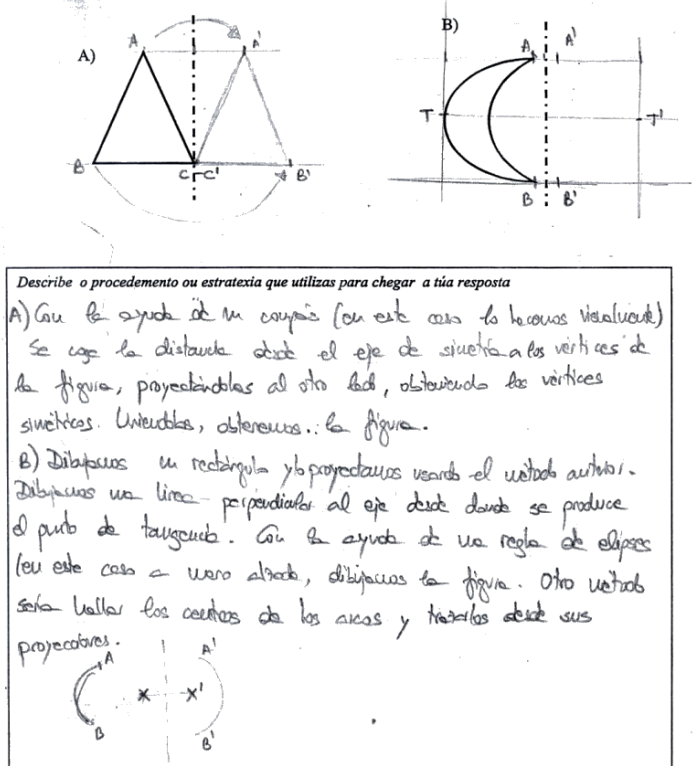

Figura 3 - Resolución del estudiante 25 Fuente: Producciones de los estudiantes.

CC2-Representación en perspectiva sin apoyo de los ejes. En este caso los alumnos representan en perspectiva, al otro lado de los ejes, los cuerpos de revolución generados mentalmente. No utilizan los ejes para construir la representación plana de los cuerpos.

CC3-Representación de una secuencia de giros en el plano. La representación gráfica asociada a esta configuración está conformada en cada caso por un conjunto de figuras congruentes a la dada, por medio de las cuales los estudiantes representan el proceso por el que llegan a la imagen mental final, que coincide con la figura de partida. La Figura 4 ejemplifica esta representación gráfica, junto con una representación escrita, donde el alumno 132 replica varias veces las figuras dadas en el plano entorno a los ejes.

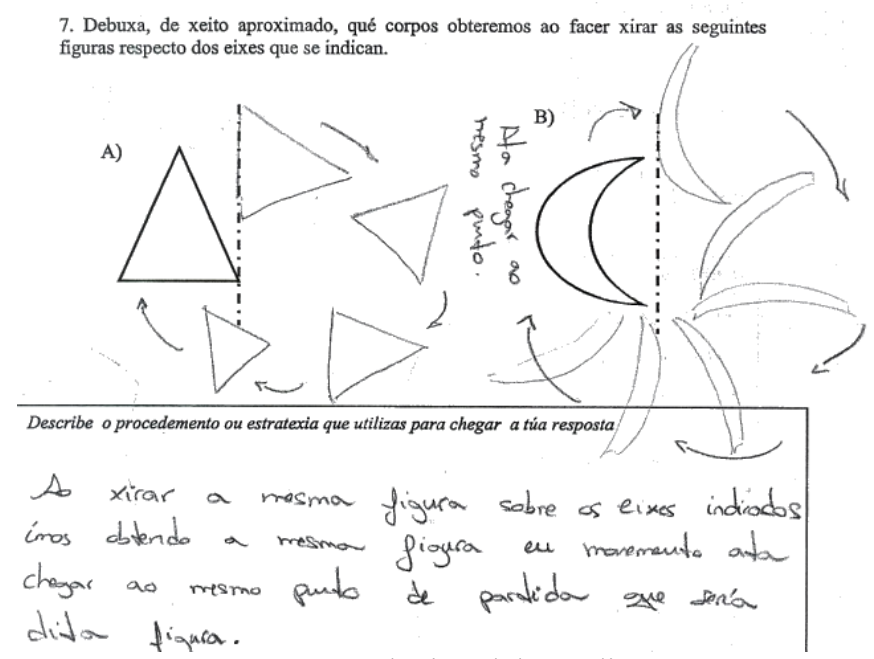

Figura 4 - Resolución del estudiante 132

Fuente: Producciones de los estudiantes.

CC4-Representación en perspectiva con apoyo de los ejes. La representación gráfica 
asociada a esta configuración consiste en dibujar las figuras simétricas a las dadas, y marcar las circunferencias (elipses en perspectiva) que se generan al rotar la figura y contienen a puntos simétricos.

CC5-Representación de la sección ortogonal-frontal. En esta configuración el procedimiento seguido para la representación plana de los cuerpos generados consiste en dibujar las figuras simétricas a las dadas y después unirlas mediante segmentos, dando lugar a la sección ortogonal-frontal de cada uno de los cuerpos de revolución generados. En el caso A esa representación plana presenta la imagen de un trapecio formado por tres triángulos isósceles congruentes, y en B dos curvas cerradas que aproximan a elipses concéntricas (Figura 5).
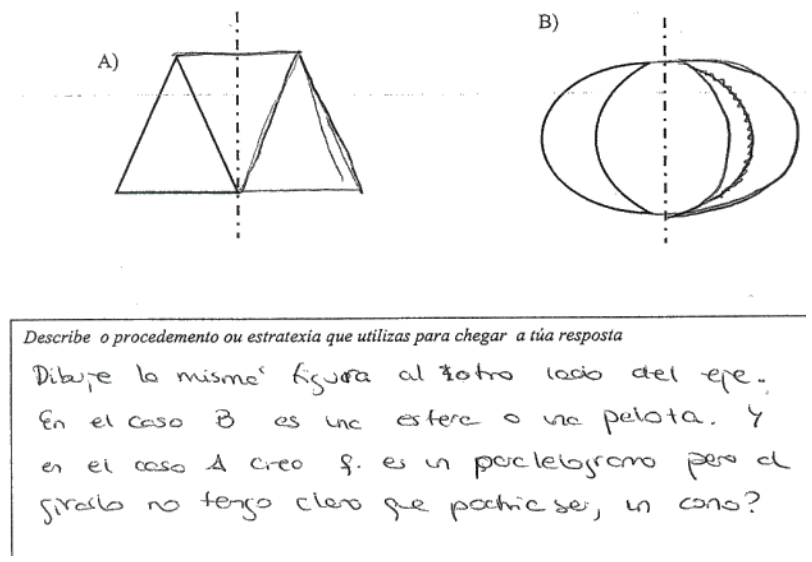

Figura 5 - Resolución del estudiante 65 Fuente: Producciones de los estudiantes

CC6-Representación escrita. La representación externa de esta configuración es verbal, describiéndose exclusivamente mediante texto escrito los cuerpos resultantes y las acciones llevadas a cabo para obtenerlos, sin apoyo de representaciones gráficas.

CC7-Representación de una secuencia de giros en el espacio. Aquí, la representación plana muestra cada una de las figuras en distintas posiciones a medida que se van girando alrededor del eje. Esta secuencia de imágenes sugiere el movimiento realizado mentalmente al hacer girar las figuras alrededor del eje. Esas imágenes se coordinan e integran dando lugar a las imágenes mentales resultantes, que serán sólidos de revolución. En la Figura 6 el alumno 25 dice obtener como resultado una pirámide para el caso A y una esfera para el B. 


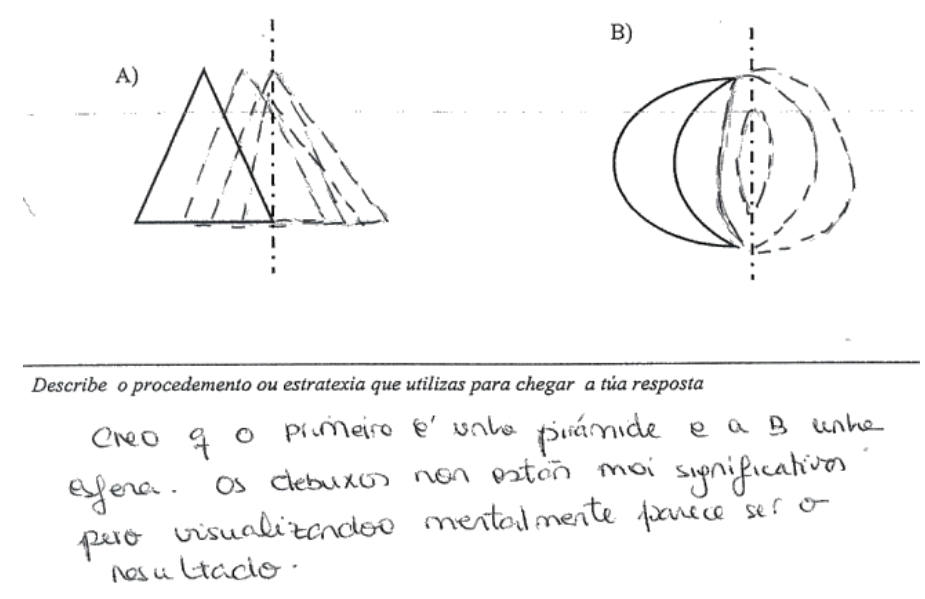

Figura 6 - Resolución del estudiante 25

Fuente: Producciones de los estudiantes

CC8- Representación de la composición giro-traslación. En este caso las imágenes mentales obtenidas son las figuras giradas $180^{\circ}$ con respecto a las dadas. Se giran las figuras dadas $180^{\circ}$ y se trasladan para que queden en línea con las figuras iniciales, pero del otro lado del eje.

CC9-Representación de la planta y alzado. La representación asociada a esta configuración se obtiene dibujando las figuras simétricas a las dadas y trazando rectas paralelas al eje que pasan por los vértices del triángulo y por los vértices de la lúnula para determinar en cada caso dos circunferencias concéntricas que representan la planta de los cuerpos de revolución generados.

\subsection{Procesamiento visual de la información}

Si se atiende al espacio donde se realiza la acción, el análisis del procesamiento visual que realizaron los alumnos de la información dada permite agrupar las configuraciones en: (1) aquellas en las que la acción se realiza en el espacio bidimensional y, por tanto, el cuerpo resultante es una figura plana; (2) aquellas en las que la acción se realiza en el espacio tridimensional, dando lugar a cuerpos tridimensionales.

En el primer grupo se encuentran las configuraciones CC1, CC3 y CC8, que suponen el $57 \%$ de la muestra. En el caso de las configuraciones CC3 y CC8, la acción a realizar es un giro y en el caso de la configuración $\mathrm{CC} 1$ una simetría. De la misma forma, las habilidades de reconocimiento de las relaciones y posiciones espaciales y la rotación mental se aplican en el plano. En este grupo, los estudiantes no integran la información del texto escrito con la representación gráfica dada en la tarea, lo que les conduce a dar como resultado figuras planas. 
En el segundo grupo están las configuraciones CC2, CC4, CC6, CC5, CC7 y CC9, que siguen el 30\% de los estudiantes. La información que extraen de las representaciones externas dadas en el enunciado de la tarea (gráfica y verbal) conduce a los estudiantes a considerar los ejes dados como ejes de rotación en el espacio y, por tanto, la acción de rotar y las habilidades espaciales implicadas se ejecutan también en el espacio. Las imágenes mentales que generan se corresponden con cuerpos de revolución y, a diferencia del anterior grupo, no hay una figura mental asociada de forma exclusiva a cada una de estas configuraciones.

Las imágenes mentales generadas por los estudiantes como consecuencia de este proceso de visualización (VPI) fueron para el caso A: cono, tronco de cono, tronco de cono sin un cono invertido, pirámide y cilindro; y para el caso B: esfera, esfera hueca, elipsoide achatado con un agujero y esfera truncada o esfera achatada.

El porcentaje de estudiantes que obtuvieron imágenes correctas no alcanzó el 9\% de la muestra y el 5\% de ellos dieron como solución figuras no identificables. El 49,17\% de los que han considerado que se generaban figuras tridimensionales han dado como cuerpo resultante en A un cono, mientras que en B el cuerpo más frecuente ha sido una esfera $(56,67 \%)$, sin especificar en ninguno de los dos casos otro tipo de características internas (huecos, agujeros).

\section{Discusión}

En este apartado se discutirán los resultados del estudio de los procesos de visualización a partir del análisis de los errores y dificultades identificados, así como del nivel de representación gráfica de las configuraciones y de su efectividad. Esto permitirá dar cuenta del grado en que los procesos de visualización fueron desarrollados por los estudiantes.

\subsection{Errores y dificultades relacionados con los procesos de visualización VPI e IFI}

A partir del análisis de los procesos de visualización, se han identificado errores y dificultades asociados a cada uno de ellos (Tabla 2). Cabe destacar el bajo porcentaje de estudiantes que dan una representación correcta de los cuerpos de revolución generados $(8,5 \%$ para el caso A y 5,25\% para el B).

Tabla 2 - Errores asociados a la tarea

\begin{tabular}{|c|c|c|c|c|c|c|}
\hline & \multirow{2}{*}{\multicolumn{2}{|c|}{ Errores y dificultades }} & \multicolumn{2}{|c|}{ Figura A } & \multicolumn{2}{|c|}{ Figura B } \\
\hline & & & Frecuencia & $\%$ & Frecuencia & $\%$ \\
\hline \multirow[t]{2}{*}{ Proceso VPI } & $\begin{array}{l}\text { Considerar el eje de } \\
\text { rotación como eje de }\end{array}$ & $\begin{array}{l}\text { Movimiento en el } \\
\text { plano }\end{array}$ & 112 & 28 & 112 & 28 \\
\hline & simetría & Movimiento fuera & 84 & 21 & 84 & 21 \\
\hline
\end{tabular}




\begin{tabular}{|c|c|c|c|c|c|}
\hline \multirow{6}{*}{ Proceso IFI } & del plano & & & & \\
\hline & No considerar la distancia al eje & 75 & 18,75 & 96 & 24 \\
\hline & Girar en el plano las figuras & 32 & 8 & 32 & 8 \\
\hline & $\begin{array}{l}\text { No coordinar diferentes direcciones en la } \\
\text { representación plana }\end{array}$ & 31 & 7,75 & 23 & 5,75 \\
\hline & Sin errores/dificultades & 34 & 8,5 & 21 & 5,25 \\
\hline & Sin respuesta & 32 & 8 & 32 & 8 \\
\hline Total & & 400 & 100 & 400 & 100 \\
\hline
\end{tabular}

Fuente: Elaboración propia

Se detectaron dos errores relacionados con el proceso VPI: (1) considerar el eje de rotación como eje de simetría, (2) girar las figuras en el plano; y una dificultad asociada a no considerar la distancia de las figuras al eje. El primer error se produce cuando los estudiantes identifican la representación gráfica de una figura y un eje a su derecha como la imagen del concepto de simetría, lo que conduce a que se asocie con dicho concepto y no interpreten bien el enunciado de la tarea. Se relaciona con la dificultad de separar un concepto geométrico de su representación externa (MESQUITA, 1992).

Este error está asociado de forma directa a la configuración CC1. Los resultados muestran dos imágenes conceptuales de simetría subyacentes, una se refiere a la simetría como movimiento rígido en el plano que guarda equidistancia de un punto y su simétrico al eje, y la otra considera la simetría como un movimiento que físicamente no se produce en el plano, sino que hay que salirse del plano para mover la figura y que se produzca un cambio de orientación (JAIME; GUTIÉRREZ, 1996).

El segundo error se deriva de la noción primaria de los estudiantes sobre los giros, que tiene que ver con la característica visual que corresponde al desplazamiento circular sobre un plano que se traza al girar (por ejemplo, las manecillas del reloj). Es un error exclusivo de la configuración CC3. No considerar la distancia al eje impide establecer correctamente la relación entre la figura y el eje de rotación (equidistancia al centro de giro). Esta dificultad se relaciona directamente con la habilidad de reconocimiento de posiciones en el espacio y provoca que no se reconozcan los huecos interiores, generados precisamente por la distancia al eje de determinados puntos en cada una de las figuras.

En relación con el proceso IFI, los alumnos manifestaron, principalmente, dificultades para describir (verbal o gráficamente) las figuras resultantes. Al analizar las descripciones escritas se encontró que los estudiantes incorporan leyendas relativas a atributos de los componentes de las figuras, al igual que en el trabajo de Hazaña y Akai (1993).

Así, por ejemplo, nombran la forma de las caras etiquetando cual es la superior, frontal etc., incluso ofreciendo, en algunos casos, descripciones verbales del sólido entero, por ejemplo, en el caso A que parece una taza y en el caso B una pelota. Ellos mismos reflejan las 
dificultades que encuentran cuando dibujan sus representaciones gráficas, indicando la confrontación existente con su imagen mental.

Al igual que ocurre en las investigaciones realizadas con estudiantes de primaria (GUTIÉRREZ, 1998a), los estudiantes para maestro son conscientes de la incorrección de sus dibujos, borrándolos y volviéndolos a hacer. Estas dificultades se traducen en errores de procedimiento a la hora de coordinar las diferentes direcciones de los segmentos que integran la representación plana. Están ligados, principalmente, a las configuraciones CC6 y CC5. Si hacemos una comparación entre las dos figuras, esta dificultad se manifiesta, en mayor medida, para A (7,75\% frente al 5,25\%), lo que puede ser debido a la falta de simetría con respecto a un eje horizontal, que provoca que haya, en la misma perpendicular, puntos a diferente distancia del eje de rotación y dificulta la creación de la imagen mental y, por tanto, su descripción verbal o representación gráfica.

\subsection{Niveles de representación gráfica y efectividad de las configuraciones}

El proceso IFI es el encargado de analizar las imágenes mentales generadas en la visualización y de expresarlas externamente a través de una nueva imagen. En la tarea propuesta en este estudio se exige una representación gráfica de los cuerpos de revolución generados, lo que conlleva que cada configuración cognitiva tenga asociada una representación de esos cuerpos, como se ha visto en la sección 4. Por otra parte, esas representaciones son, como ya se ha comentado, la principal evidencia que se tiene de cómo son las imágenes de los cuerpos generados por los estudiantes.

Esto sugiere estudiar si es posible establecer algún tipo de jerarquía en las configuraciones en función de la representación gráfica asociada. Se han utilizado para ello los niveles de sofisticación en la representación de formas tridimensionales que proponen Pittalis, Mousoulides y Christou (2009).

En las configuraciones $\mathrm{CC} 1, \mathrm{CC} 3, \mathrm{CC} 8$ la atención se centra en asociaciones visuales con los ejemplos más populares, aquellos que existen en la imagen del concepto de todos los estudiantes (HERSHKOWITZ, 1990), en este caso la imagen del concepto de simetría. Algunos de los estudiantes establecen asociaciones verbales de una palabra con su significado más habitual, en esta tarea en la palabra giro. Las habilidades de reconocimiento de las relaciones y posiciones espaciales y de rotación se aplican al plano y no al espacio. Por tanto, no cabe aquí establecer ningún paralelismo con los niveles de Pittalis et al. (2009) ya que no hay cuerpos tridimensionales que representar. 
En la configuración CC6 se ponen en funcionamiento las habilidades de rotación mental, reconocimiento de posiciones en el espacio y conservación de las relaciones espaciales; pero no se utilizan convenciones de representaciones planas de objetos tridimensionales para representar gráficamente dichos cuerpos. La dificultad se centra en la comunicación gráfica del proceso IFI, pero las imágenes mentales creadas pueden ser correctas. No se encuentran estudiantes de nivel 1, según la clasificación de Pittalis, Mousoulides y Christou (2009), debido a que los estudiantes que siguen esta configuración, donde la representación externa es verbal (escrita), son conscientes de que sus dibujos no representarían los objetos y de que es necesario coordinar caras y diferentes vistas de los cuerpos tridimensionales, aunque no los sepan dibujar.

En la configuración CC5 la representación de los cuerpos tridimensionales generados se presenta únicamente por una de las vistas ortogonales. El tipo de representación realizado necesita apoyo verbal para comunicar la estructura completa de dichos cuerpos. Alguna de esas representaciones gráficas consiste en representar el cono como un triángulo con una cara curva únicamente, sin más convenciones, y una esfera como circunferencia. Esas representaciones gráficas sitúan a los estudiantes en el nivel 2 de Pittalis, Mousoulides y Christou (2009).

Las configuraciones $\mathrm{CC} 2$ y CC7 utilizan convenciones para el dibujo de objetos tridimensionales, sin embargo, no se indican huecos ni otras características internas de las figuras, lo que no da una información completa de las imágenes creadas. No se consigue dotar plenamente a los cuerpos de profundidad y por ello, en la mayor parte de los casos, el tipo de representación es mixto, necesitando apoyo verbal o escrito para comunicar la estructura del cuerpo generado. El tipo de representación se corresponde con el nivel 3 de Pittalis, Mousoulides y Christou (2009).

En las configuraciones CC4 y CC9 se implementan las convenciones para representaciones bidimensionales de cuerpos tridimensionales, indicando elementos de la estructura interna y externa de los cuerpos. La comunicación de la información sobre las imágenes mentales creadas es bastante clara, lo que manifiesta que las habilidades de reconocimiento de posiciones y relaciones espaciales y la de rotación mental están bastante desarrolladas. El tipo de representación corresponde al nivel 4 de Pittalis, Mousoulides y Christou (2009).

Para aquellas configuraciones cognitivas susceptibles de producir resultados correctos (CC2, CC4, CC5, CC6, CC7 y CC9), podemos considerar su efectividad, entendida como el porcentaje de resultados correctos en la resolución de la tarea por los estudiantes que siguieron esa configuración (GORGORIÓ, 1998).

En la Tabla 3 se presenta la relación ente los niveles de representación gráfica de las 
configuraciones cognitivas y la efectividad de estas. Se observa que a mayor nivel de representación mayor efectividad de las configuraciones. Los resultados muestran que la configuración CC2, la de mayor frecuencia (17\%), tiene una efectividad poco relevante.

Por otro lado, la configuración menos frecuente, la CC9, tiene mayor efectividad, lo que puede deberse a que los estudiantes con los que se asocia están habituados al dibujo técnico y a trabajar con diferentes puntos de vista, como muestran el lenguaje y los códigos de representación plana que han utilizado. Lo anterior se traduce en que la parte del proceso IFI que se ocupa de expresar la información sobre los sólidos generados fue determinante para resolver la tarea con éxito.

Tabla 3 - Relación entre el nivel de representación en las CC y la efectividad

\begin{tabular}{|c|c|c|c|}
\hline Configuración & Tipo de representación & Nivel de representación & $\%$ \\
\hline CC9 & Representación de planta y alzado & 4 & 80 \\
\hline $\mathrm{CC} 4$ & $\begin{array}{l}\text { Representación en perspectiva con apoyo de } \\
\text { los ejes }\end{array}$ & 4 & 38,10 \\
\hline $\mathrm{CC} 7$ & $\begin{array}{l}\text { Representación de una secuencia de giros en } \\
\text { el espacio }\end{array}$ & 3 & 10 \\
\hline $\mathrm{CC} 2$ & $\begin{array}{l}\text { Representación en perspectiva sin apoyo de } \\
\text { los ejes }\end{array}$ & 3 & 3,03 \\
\hline $\mathrm{CC} 5$ & Representación de la sección ortogonal-frontal & 2 & 0 \\
\hline CC6 & Representación escrita & -- & 0 \\
\hline
\end{tabular}

Fuente: Elaboración propia

\section{Conclusiones}

En este trabajo se han analizado los dos procesos implicados en la visualización, el procesamiento visual de la información (VPI) y el de interpretación de la información figurativa (IFI), en una tarea que requiere generar y representar cuerpos de revolución. La tarea se ha aplicado a una muestra de cuatrocientos futuros maestros de Educación Primaria.

Se ha utilizado como herramienta analítica la noción de configuración ontosemiótica, en su vertiente epistémica y cognitiva. Esto ha permitido identificar nueve configuraciones cognitivas, cada una de las cuales describe la manera particular en la que los estudiantes pusieron en juego esos dos procesos. El análisis de los procesos de visualización, a la luz de la herramienta analítica, ha contribuido a apuntar errores y dificultades de los estudiantes al enfrentarse a una tarea de generación de sólidos de revolución, así como a esbozar niveles de representación para este caso.

Para el proceso VPI, los resultados muestran que el 49,50\% de los estudiantes (configuraciones $\mathrm{CC} 1, \mathrm{CC} 3$ y CC8) asocian visualmente la imagen presentada en el enunciado de la tarea con prototipos. En este caso, con la representación estándar de una simetría o un giro 
en el plano, y por tanto dan como resultado figuras planas.

Para las restantes configuraciones, se observa que todas requieren movilizar las habilidades de visualización necesarias para resolver la tarea: rotación mental y reconocimiento de relaciones y posiciones espaciales. En particular, la mayoría de los estudiantes ponen en funcionamiento la primera de ellas, sin embargo, tienen dificultades para movilizar las otras dos habilidades, como se constata en el porcentaje de estudiantes que no establecen correctamente la relación entre la figura y el eje de rotación (18,75\% en A y $24 \%$ en B).

Para el proceso IFI, centrándonos en aquellas configuraciones que crearon cuerpos de revolución, los tipos de representaciones planas asociadas a esas configuraciones fueron las siguientes: representación en perspectiva (configuraciones $\mathrm{CC} 2$ y CC4); representación de planta y alzado (CC9); representación de la sección ortogonal-frontal (CC5); y la representación de una secuencia de giros en el espacio (CC7).

Todas ellas deberían seguir códigos y claves de representación sin los cuales no se puede hacer una lectura correcta de las mismas. Por ejemplo, en la configuración CC4, dibujar las circunferencias de las bases de las figuras planas es un código que confiere a la estructura un efecto tridimensional. En el caso particular de la configuración CC5, los códigos son insuficientes y por ello la representación gráfica asociada a esta configuración se suele acompañar de un discurso escrito. A pesar de que la representación en perspectiva es la más frecuente $(21,75 \%)$, es la más difícil de realizar con corrección.

Los resultados generales del estudio muestran un porcentaje excesivamente bajo de estudiantes que fueron capaces de realizar la tarea completa correctamente $(5,25 \%)$. Se puede concluir que los estudiantes se limitan a ajustar las imágenes conceptuales creadas a los prototipos que conocen, sin analizar las características y propiedades geométricas de los mismos; siendo los cuerpos con mayor representatividad el cono $(49,17 \%)$ y la esfera $(60,83 \%)$. Los errores relacionados con el proceso VPI tienen un peso significativo en la resolución de la tarea (suponen más del 75\% tanto para A como para B), destacando el error de considerar el eje de rotación como eje de simetría y el error de no considerar la distancia al eje.

Además, el análisis de la efectividad de las configuraciones muestra que la parte del proceso IFI que se ocupa de expresar la información sobre los sólidos generados fue determinante para resolver la tarea con éxito, ya que, a mayor nivel de representación, siguiendo los niveles de sofisticación en la representación de objetos tridimensionales de Pittalis, Mousoulides y Christou (2009), mayor efectividad de las configuraciones cognitivas.

No obstante, debemos tomar esto con precaución, pues las representaciones utilizadas en $\mathrm{CC} 9$ y CC4 se encuentran en el mismo nivel, pero la diferencia de efectividad es alta. Por 
otra parte, seis de las configuraciones permitirían llegar a la solución correcta, pero dificultades de los estudiantes con las habilidades de reconocimiento de posiciones en el espacio y con el proceso IFI lo impiden.

La herramienta analítica ha permitido, por tanto, detectar y especificar cómo realizan los estudiantes los dos procesos implicados en la visualización y en dónde se encuentran las principales dificultades y errores relacionados con dichos procesos. La dirección a seguir para la formación de los futuros maestros con relación a este contenido parece apuntar hacia actividades que movilicen habilidades de visualización como el reconocimiento de posiciones y relaciones espaciales y la rotación espacial, y hacia el tratamiento de códigos que permitan comunicar las imágenes creadas a través de una representación externa (gráfica o verbal).

\section{Agradecimientos}

Trabajo financiado por el proyecto STEM4youth: Promotion of Stem Education by Key Scientific Challenges and their Impact on our Life and Career Perspectives, Programa Horizon 2020 (H2020- Seac-2015-1-710577) y por FEDER/Ministerio de Ciencia, Innovación y Universidades - Agencia Estatal de Investigación/_Proyecto EDU2017-84979-R.

\section{Referencias}

BEN-CHAIM, D.; LAPPAN, G.; HOUANG, R. T. The effect of instruction on spatial visualization skills of middle school boys and girls. American Educational Research Journal, Thousand Oaks, California, v. 25, n. 1, p. 51-71, Jan. 1988.

BISHOP, A. J. Review of research on visualisation in mathematics education. Focus on Learning Problems in Mathematics, Framingham, Mass., US, v. 11, n. 1, p. 7-16, Win-Spr. 1989.

BLANCO, T. F. Una aproximación ontosemiótica a la visualización y el razonamiento espacial. 2011.Tesis (Doctorado en Didáctica de las Ciencias Experimetales y de la Matemática) - Facultade de Ciencias da Educación, Universidade de Santiago de Compostela, Santiago de Compostela, 2011.

CLEMENTS, M. Fifty Years of Thinking About Visualization and Visualizing in Mathematics Education: A Historical Overview. In: FRIED, M. N.; DREYFUS, T. (Ed.). Mathematics \& Mathematics Education: Searching for Common Ground. Dordrecht: Springer, 2014. p. 177-192. Disponibel en: <http://doi.org/10.1007/978-94-007-7473-5_11>. Accesso em: Enero de 2018.

COHEN, N. Curved solid nets. In: INTERNATIONAL CONFERENCE OF PSYCHOLOGY IN MATHEMATICS EDUCATION, 27., 2003, Honolulu. Proceedings... Honolulu: PME, 2003. p. 229236.

DEL GRANDE, J. Spatial sense. Arithmetic Teacher, Reston, VA, v. 37, n. 6, p. 14-20, Feb.1990.

DEL GRANDE, J. Spatial perception and primary geometry. In: MONTGOMERY, M., SHULTE, A. (Ed.). Learning and Teaching geometry, K-12. Reston, VA: National Council of Teachers of Mathematics, 1987. p. 127-135. 
FONT, V.; GODINO, J. D.; GALLARDO, J. The emergence of objects from mathematical practices. Educational Studies in Mathematics, Netherlands, v. 82, n.1, p. 97-124, Jan. 2013.

GAULIN, C. The need for emphasizing various graphical representations of 3-dimensional shapes and relations. In: INTERNATIONAL CONFERENCE OF PSYCHOLOGY IN MATHEMATICS EDUCATION, 9., 1985, Noordwijkehout. Proceedings... Noordwijkehout: OW \& OC, State University of Utrecht, 1985. p. 53-71.

GODINO, J. D. Perspectiva ontosemiótica de la competencia y comprensión matemática. La matematica e la sua didattica, Bologna, v. 4, p. 434-450, 2002.

GODINO, J. D.; BATANERO, C.; FONT, V. The onto-semiotic approach to research in mathematics education. ZDM. The International Journal on Mathematics Education, Karlsruhe, v. 39, n .1-2, p. 127 - 135, Jan. 2007. doi:10.1007/s11858-006-0004-1

GODINO, J. D. et al. Synergy between visual and analytical languages in mathematical thinking. In: CONGRESS OF THE EUROPEAN SOCIETY FOR RESEARCH IN MATHEMATICS EDUCATION, 8., 2013, Manavgat-Side/Antalya. Proceedings... Ankara: Middle East Technical University and ERME, 2013. p. 645-654.

GODINO, J. D.; FONT, V.; WILHELMI, M. R. Análisis didáctico de procesos de estudio matemático basado en el enfoque ontosemiótico. Publicaciones, Melilla, v. 38, p. 25-48, abr. 2008.

GODINO, J. D. et al. Una aproximación ontosemiótica a la visualización en educación matemática. Enseñanza de las ciencias: revista de investigación y experiencias didácticas, Barcelona, v. 30, n. 2, p. 109-130, set. 2012.

GODINO, J. D. et al. Evaluación de Conocimientos Didáctico-Matemáticos sobre Visualización de Objetos Tridimensionales en Futuros Profesores de Educación Primaria. REDIMAT - Journal of Research in Mathematics Education, Barcelona, v. 5, n. 3, p. 235-262, Oct. 2016.

GONZATO, M.; BLANCO, T. F.; GODINO, J.D. Tareas para el desarrollo de habilidades de visualización y orientación espacial: un estudio sistemático basado en la investigación didáctica. Números, Tenerife, v. 77, p. 99-117, jul. 2011.

GONZATO, M.; GODINO, J. D.; CONTRERAS, J. M. Evaluación de conocimientos sobre la visualización de objetos tridimensionales en maestros en formación. En: MARÍN, M.; FERNÁNDEZ, G.; BLANCO, L. J.; PALAREA, M (Ed.). Investigación en Educación Matemática XV. Ciudad Real: SEIEM, 2011. p. 383-392.

GORGORIÓ, N. Exploring the functionality of visual and non-visual strategies in solving rotation problems. Educational Studies in Mathematics, Netherlands, v. 35, p. 207-231, Mar. 1998.

GUILLÉN, G.; GONZÁLEZ, E.; GARCÍA, M.A. Criterios específicos para analizar la geometría en libros de texto para la enseñanza primaria y secundaria obligatoria. Análisis desde los cuerpos de revolución. En: GONZÁLEZ, M.J.; GONZÁLEZ, M.T.; MURILLO, J. (Ed.). Investigación en Educación Matemática XIII. Santander: SEIEM, 2009. p. 247-258.

GUILLÉN, G. et al. La enseñanza de la geometría de los sólidos en EGB. 1992. Memoria final de proyecto de investigación. Institución Valenciana de Estudios e Investigación «Alfonso el Magnánimo», Valencia, 1992.

GUTIÉRREZ, A. Las representaciones planas de cuerpos 3-dimensionales en la enseñanza de la geometría espacial. Revista EMA, Santafé de Bogotá, Colombia, v. 3., n. 3, p. 193-220, 1998 a. 
GUTIÉRREZ, A. Tendencias actuales de investigación en geometría y visualización. Manuscrito de conferencia invitada en el "Encuentro de Investigación en Educación Matemática", TIEM-98. Centre de Recerca Matemàtica, Institut d'Estudis Catalans, Barcelona, 1998b.

GUTIÉRREZ, A. Visualization in 3-dimensional geometry: In search of a framework. In: PUIG, L.; GUTIÉRREZ, A. (Ed.). PME INTERNATIONAL CONFERENCE, 20., 1996, Valencia.

Proceedings... Valencia: PME, 1996a. p. 3-19.

GUTIÉRREZ, A. Children's ability for using different plane representations of space figures. In: BATTURO, A.R. (Ed.). New directions in geometry education. Brisbane: Centre for Math. and Sc. Education, Q.U.T., 1996b. p. 33-42.

GUTIÉRREZ, A. Exploring the links between van Hiele levels and 3-dimensional geometry. Structural topology, Montréal, v. 18, p. 31-48, Jan. 1992.

HAZAMA, S.; AKAI, T. Pupil's development of graphical representations of 3-dimensional figures: On technical difficulties, conflicts or dilemmas, and controls in the drawing process. In: PME INTERNATIONAL CONFERENCE, 17., 1993, Tsukuba. Proceedings... Tsukuba: PME, 1993. p. 161-168.

HART, L. C. et al. An examination of research methods in mathematics education. Journal of Mixed Methods Research, London, v. 30, p. 26-41, Jan. 2009.

HERSHKOWITZ, R. Psychological aspects of learning geometry. In: NESHER, P.; KILPATRICK, P. AND J. (Ed.). Mathematics and cognition: A research synthesis by the International Group for the psychology of mathematics education. Cambridge: Cambridge U.P., 1990. p. 70-95.

HERSHKOWITZ, R. et al. Space and shape. In: BISHOP, A. J. ET AL. (Ed.). International Handbook of Mathematics Education. London: Kluwer, 1996. p. 161-204.

JAIME, A., GUTIÉRREZ, A. El grupo de las isometrías del plano. Educación Matemática en Secundaria, v. 13. Madrid: Síntesis, 1996.

KOPELMAN, E.; VINNER, S. Visualization and reasoning about lines in space: school and beyond. In: PONTE, J. P.; MATOS, J. F. (Ed.). PME INTERNATIONAL CONFERENCE, 18., 1994, Lisbon. Proceedings... Lisbon: PME, 1994. p. 97-103.

LUBINSKI, D. Spatial ability and STEM: A sleeping giant for talent identification and development. Personality and Individual Differences, Elsmford, N. Y., v. 49, n. 4, p. 344-351, Sep. 2010.

MESQUITA, A.L. The types of apprehension in spatial geometry: sketch of a research. Structural topology, Montréal, v. 18, p. 19-30, Jan. 1992.

MITCHELMORE, M.C. Prediction of developmental stages in the representation of regular space figures. Journal for Research in Mathematics Education, Reston, VA, v. 11, n. 2, p. 83-93, Mar. 1980a.

MITCHELMORE, M.C. Three dimensional geometrical drawing in three cultures. Educational studies in Mathematics, Netherlands, v. 11, p. 205-216, May $1980 \mathrm{~b}$.

MITCHELMORE, M.C. "Geometry and spatial learning: Some lessons from Jamaican experience. For the learning of mathematics, Fredericton, NB, v. 3, n. 3, p. 2-7, Nov. 1983.

PARZYSZ, B. "Knowing" vs "seeing". Problem of the plane representation of space geometry figures. Educational Studies in Mathematics, Netherlands, v. 19, p. 79-92, Feb.1988. 
PITTALIS, M.; MOUSOULIDES, N.; CHRISTOU, C. Level of sophistication in representing 3D shapes. In: PME INTERNATIONAL CONFERENCE, 33., 2009, Thessalonki. Proceeding... Thessaloniki: PME, 2009. p. 385-392.

Submetido em 13 de Março de 2018. Aprovado em 03 de Dezembro de 2018. 